\title{
Adaptive limited feedback for intercell interference cancelation in cooperative downlink multicell networks
}

\author{
Berna Özbek ${ }^{1,2}$, Didier Le Ruyet ${ }^{1}$ \\ ${ }^{1}$ Electronics and Communications Laboratory, CNAM, 292 rue Saint Martin, 75141 Paris, France \\ ${ }^{2}$ Electrical and Electronics Eng. Dep., Izmir Institute of Technology, Urla, 35430, Izmir, Turkey \\ bernaozbek@iyte.edu.tr,didier.le_ruyet@cnam.fr
}

\begin{abstract}
In this paper, we evaluate intercell interference cancelation techniques in cooperative downlink multicell systems. We compare two classical beamforming strategies for the design of the beamforming vectors: maximum ratio combining precoding and partial zero-forcing. Since full channel state information at the transmission is unfeasible, we consider a limited feedback link and compare different strategies for bit partitioning of the feedback information for serving and interferer base stations. We propose a simple adaptive bit partitioning strategy to select an optimum transmission scheme at the base station. We show that the adaptive bit portioning algorithm is improved the sum capacity performance of the multicell networks significantly.
\end{abstract}

\section{INTRODUCTION}

Coordinated multicell transmission has recently received significant attention since it can increase data rates by mitigating intercell interference (ICI) [1]. Full cooperation between the base stations (BSs) also called network multiple input multiple output (MIMO) scheme transforms the interference channel into a MIMO broadcast channel. Dirty paper coding and sub-optimal linear beamforming such as Zero Forcing (ZF) have been investigated in [2] [3] and upper bounds on the sum rates have been obtained for multicell transmission. Assuming that a linear transceiver processing is employed, a base station (BS) equipped with $N_{t}$ antennas is able to accommodate up to $N_{t}-1$ interference signals in a coordinated multicell transmission.

Coordinated multicell transmission requires channel state information (CSI) of all jointly processed links. A large amount of data needs to be exchanged between the BSs. For example, the requirement for CSI grows in proportion to the number of transmit antennas, the number of BSs and the number of users. In order to reduce the load on the backhaul links, the partial cooperative strategies have been considered in [4]. In coordinated single-cell transmission, the BSs share only CSI and no data exchange is required where a part of ICI is removed. Besides, it is possible to apply different beamforming strategies such as Maximum Ratio Combining (MRC) and Partial Zero-Forcing (PZF) beamforming [5] by removing some ICI.

In [6], the authors have considered ICI cancelation for the mitigation of other cell interference. They have proposed an adaptive strategy where the BSs exchange the position of their users and then select the best transmission strategy between MRC and PZF. They have also shown that the adaptive strategy can provide significant gain compared to the schemes without ICI. In [7], a limited feedback scenario is considered for a multicell beamforming approach that approximately maximizes the sum-rates in a two-cell two-user network by quantizing the CSI of serving and interfering base station with the usage of two separate codebooks. In [8], a chordal distance-based compression scheme is introduced to reduce the feedback overhead and combat the intercell interference.

In this paper, we propose a simplified bit allocation algorithm to transfer CSI of the users for limited feedback link to reduce the backhaul load. In the proposed algorithm, selection of the transmission strategies is only dependent of the position of the user in the multicell network rather than the position of the users in other cells. We demonstrate that the proposed algorithm outperforms the uniform bit allocation scheme for feedback link.

This paper is organized as follows. In Section II, we describe the system model for the coordinated single cell transmission including limited feedback links. In Section III, we present the proposed algorithm to select transmission strategies and bit partitioning schemes for feedback links. In Section IV and V, we illustrate the performance results and draw the concluding remarks of this paper respectively.

\section{System Model}

We consider a downlink multicell wireless network with $K$ cells. As shown in Figure 1, the base station at each cell is composed of $N_{t}$ transmit antennas and each user is equipped with one single antenna.

Using a narrow band flat-fading model, the baseband received signal for the user in the $i^{t h}$ cell is written as,

$$
y_{i}=\sqrt{P_{i, i}} \mathbf{h}_{i, i}^{H} \mathbf{f}_{i} x_{i}+\sum_{j \neq i} \sqrt{P_{i, j}} \mathbf{h}_{i, j}^{H} \mathbf{f}_{j} x_{j}+n_{i}
$$

where $P_{i, j}$ is the received power at the $i^{t h}$ user from the $j^{\text {th }}$ $\mathrm{BS}, \mathbf{h}_{i, j}$ is the channel between the $j^{\text {th }} \mathrm{BS}$ and the user of the $i^{\text {th }}$ cell. $\mathbf{f}_{i}$ is the beamforming vector at the $i^{\text {th }}$ base station and $n_{i}$ is the complex additive zero mean white Gaussian noise 


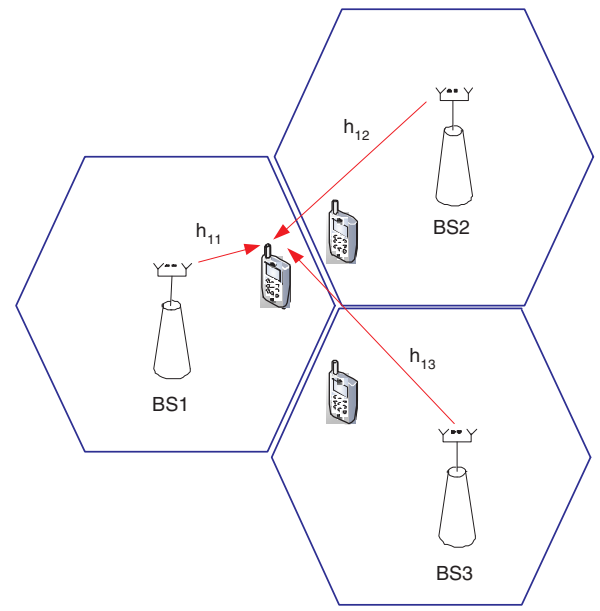

Fig. 1. A multicell system with $K=3$ base stations having $N_{t}$ transmit antennas.

at the receive antenna with $\mathbb{E}\left|n_{i}\right|^{2}=1$. It is assumed that each component of $\mathbf{h}_{i, j}$ has independent identically distributed random variable with $\mathcal{C N}(0,1)$ and $\mathbb{E}\left\{\left\|x_{i}\right\|^{2}\right\}$ is normalized to 1 .

The instantaneous signal-to-interference-plus-noise ratio (SINR) for the user of the $i^{t h}$ cell is given by:

$$
\operatorname{SINR}_{i}=\frac{P_{i, i}\left|\mathbf{h}_{i, i}^{H} \mathbf{f}_{i}\right|^{2}}{1+\sum_{j \neq i} P_{i, j}\left|\mathbf{h}_{i, j}^{H} \mathbf{f}_{j}\right|^{2}}
$$

The associated average rate at each cell and the sum rate for multicell system are respectively calculated as

$$
R_{i}=\mathbb{E}\left[\log _{2}\left(1+\mathrm{SINR}_{i}\right)\right], \quad R=\sum_{i=1}^{K} R_{i}
$$

\section{A. Transmission Strategies}

In this work, we consider two classical transmission strategies for the design of the beamforming vectors $\mathbf{f}_{i}$.

1) Maximum Ratio Combining (MRC) beamforming: The other cell interference is ignored and the precoding vector is designed according to the channel direction of the user itself. For the $i^{t h} \mathrm{BS}$, the precoding vector is given by,

$$
\mathbf{f}_{i}=\mathbf{h}_{i, i} /\left\|\mathbf{h}_{i, i}\right\|
$$

The distribution of $\left|\mathbf{h}_{i, i}^{H} \mathbf{f}_{i}\right|^{2}$ for MRC precoding is given by,

$$
\left|\mathbf{h}_{i, i}^{H} \mathbf{f}_{i}\right|^{2} \sim \chi_{2 N_{t}}^{2}
$$

where $\chi_{n}^{2}$ denotes the chi-square random variable with $n$ degrees of freedom.

\section{2) Partial Zero-Forcing (PZF) beamforming:}

In order to maximize $\left|\mathbf{h}_{i, i}^{H} \mathbf{f}_{i}\right|^{2}$, some degrees of freedom are used for the ICI cancelation. This corresponds to select $\mathbf{f}_{i}$ in the direction of the projection of the channel vector $\mathbf{h}_{i, i}$ on the nullspace of $\mathbf{H}_{i}=\left[\mathbf{h}_{1, i}, \ldots, \mathbf{h}_{i-1, i}, \mathbf{h}_{i+1, i}, \ldots, \mathbf{h}_{K, i}\right]$. The unnormalized version of the precoding vector is given by

$$
\tilde{\mathbf{f}}_{i}=(\mathbf{I}-\mathbf{P}) \mathbf{h}_{i, i}
$$

where $\mathbf{P}=\mathbf{H}_{i}\left(\mathbf{H}_{i}^{H} \mathbf{H}_{i}\right)^{-1} \mathbf{H}_{i}^{H}$ which is the projection matrix on $\mathbf{H}_{i}$.
For PZF precoding, the distribution of $\left|\mathbf{h}_{i, i}^{H} \mathbf{f}_{i}\right|^{2}$ [5] is given by,

$$
\left|\mathbf{h}_{i, i}^{H} \mathbf{f}_{i}\right|^{2} \sim \chi_{2\left(N_{t}-(K-1)\right)}^{2}
$$

\section{B. Limited feedback link}

In order to address the lack of perfect CSI, a classical solution is to quantize the channel direction information (CDI) and the channel quality information (CQI) before transmission over the finite rate feedback link. The CDI codebook is known by both the user side and the BSs. Each user quantizes its CDI to the closest codeword. At the BS, CDI should be constructed by minimizing the maximum inner product between codewords [9]. In order to analyze the achievable average rate, we use random vector quantization (RVQ) since the optimal vector quantizer is not known in general. For $\mathrm{RVQ}$, each vector is independently chosen from the isotropic distribution on the $N_{t}$-dimensional unit sphere [10].

Firstly, we consider a limited feedback link constructed for 2-cells network. Under this condition, we have four different transmission strategies at the BSs. The base station 1 (BS1) and base station 2 (BS2) can select MRC or PZF depending on the position of the user in the cell.

Depending on the chosen transmission strategy for 2-cells network, the average rate of user 1 for a fixed position $\left(x_{1}, y_{1}\right)$ is calculated as,

$$
\begin{aligned}
& R_{1}\left(P_{1,1}, P_{1,2}\right)=
\end{aligned}
$$

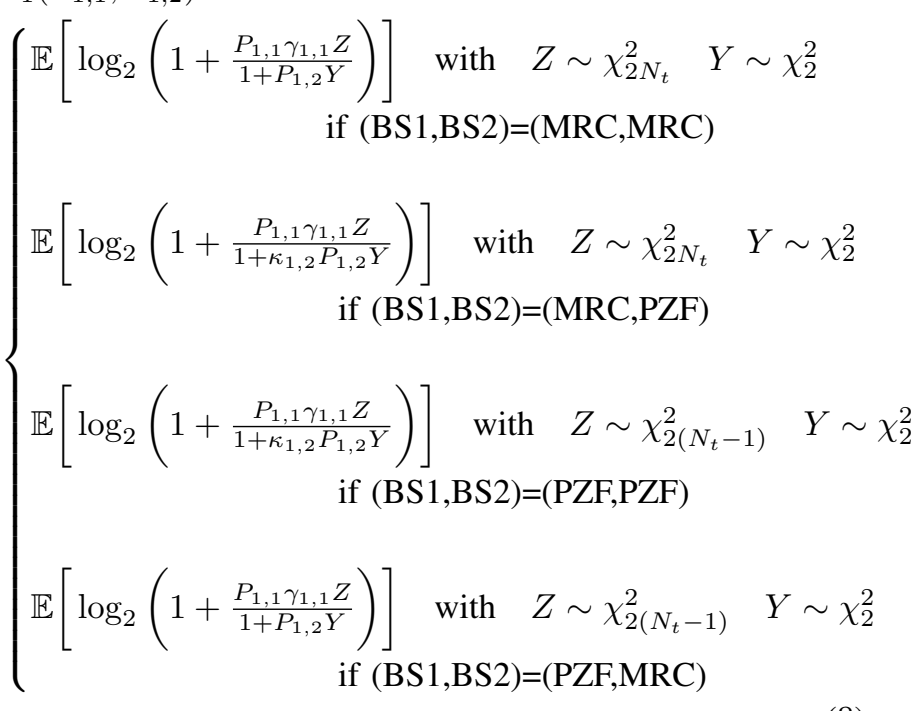

Assuming that RVQ is used [10] [11], we have:

$$
\gamma_{1,1}=1-2^{B_{1,1}} \beta\left(2^{B_{1,1}}, \frac{N_{t}}{N_{t}-1}\right)
$$

and

$$
\kappa_{1,2}=2^{B_{1,2}} \beta\left(2^{B_{1,2}}, \frac{N_{t}}{N_{t}-1}\right)
$$

where $\beta(x, y)$ is the Beta function and $B_{i, j}$ is the number of bits used for the quantization of the normalized channel vector $\mathbf{h}_{i, j}$. 
We use a path loss model where $P_{i, j}=P_{0}\left(R / d_{i, j}\right)^{\alpha}$ with $P_{0}$ is the received power at the distance $R$ from the BS (received power at the edge of the cell), $\alpha$ is the path loss exponent and $d_{i, j}$ is the distance between the $i^{t h}$ user and the $j^{\text {th }} \mathrm{BS}$.

This 2-cells network can be easily extended to the multicell case by introducing the interference from other cells.

\section{THE PROPOSED ALGORITHM FOR COOPERATIVE MULTICELL SYSTEMS}

In this section, we propose an algorithm for cooperative multicell networks to perform optimum transmission strategies with limited feedback link. The feedback link is established to send back the CDI of both the serving and the interfering BSs to the serving BSs. The total number of bits of the $i^{t h}$ user is fixed to $B_{\max }$ and we have $\sum_{j, j \neq i} B_{i, j}$ bits to send the interfering BSs information using backhaul.

In order to perform MRC or PZF beamforming at the BSs, we propose to choose the number of allocated bits for serving and interfering BSs for each user at each cell without cooperation between the BSs. The proposed algorithm is based on the definition of regions and subregions depending on the power at the cell edge and the location of the user within the multicell network.

We first split each cell's region, $\mathcal{R}_{\text {cell }}$, into two regions as $\mathcal{R}_{\text {Inner }}$ and $\mathcal{R}_{\text {Outer with }} \mathcal{R}_{\text {Inner }} \cup \mathcal{R}_{\text {Outer }}=\mathcal{R}_{\text {cell }}$. The border of the regions depends on $P_{0}$ and the number of interfering BSs. When low $P_{0}$ values are considered, there is only one region as $\mathcal{R}_{\text {Inner }}=\mathcal{R}_{\text {cell }}$ while $P_{0}$ value is high, only the PZF region is defined as $\mathcal{R}_{\text {Outer }}=\mathcal{R}_{\text {cell }}$.

While the proposed algorithm is suboptimal, we will show in the simulation results section that the performance loss is limited compared to the optimal transmission strategy where the feedback bits are selected assuming the knowledge of all the users' position.

The proposed algorithm for 2-cells networks:

For the $k^{t h}$ user:

- Step 1: The region of the user (inner/outer) is chosen according to the position of the user :

$$
U_{k}=\left(x_{k}, y_{k}\right) \in \mathcal{R}_{\text {Inner }} \quad \text { or } \quad U_{k}=\left(x_{k}, y_{k}\right) \in \mathcal{R}_{\text {Outer }}
$$

After the determination of the region of the user, the repartition of the bits from the $k^{t h}$ user to the serving and/or interfering BSs is selected in Step 2.

- Step 2a: If the user is inside the inner region, $U_{k} \in$ $\mathcal{R}_{\text {Inner }}$, all bits are assigned to the serving BS as,

$$
B_{S}=B_{k, k}=B_{\max } \quad \text { and } \quad B_{I}=B_{k, k^{\prime}}=0
$$

for $k^{\prime}=1,2, \ldots, K$ and $k^{\prime} \neq k$.

Since the user is in the inner region, the effect of the interference power coming from other BSs is ignored for the user $k$.

- Step $2 b$ : If the user is in the outer region, $U_{k} \in \mathcal{R}_{\text {Outer }}$, it is necessary to apply ICI schemes. Therefore, a portion of the bits is assigned to the serving BS and the remaining bits are reserved for the interferer BSs.
We propose to perform an adaptive bit partitioning according to the location of the user in the cell by defining subregions as $\mathcal{R}_{P Z F_{1}} \cup \mathcal{R}_{P Z F_{2}} \ldots \cup \mathcal{R}_{P Z F_{L}}=\mathcal{R}_{\text {Outer }}$ with $L$ is the number of subregions in the outer region.

- Bit selection strategy for serving BS: if the user is in the $\ell^{t h}$ subregion as $U_{k} \in \mathcal{R}_{P Z F_{\ell}}$, the predefined number of bits, $B_{k, k}$, is assigned to the $k^{\text {th }}$ serving BS.

- Bit selection strategy for interferer BS: the remaining $B_{\max }-B_{k, k}$ bits are assigned to the interferer BSs.

\section{Theoretical analysis for 2-cells networks:}

For 2-cells networks, we can compute the average rate $R_{1}$ theoretically as follows:

$$
\begin{aligned}
& R_{1}\left(P_{11}, P_{12}\right)= \\
& \left\{\begin{array}{c}
Q \mathbb{E}\left[\begin{array}{c}
\left.\log _{2}\left(1+\frac{P_{1,1} \gamma_{1,1} Z}{1+P_{1,2} Y}\right)\right]+(1-Q) \mathbb{E}\left[\log _{2}\left(1+\frac{P_{1,1} \gamma_{11} Z}{1+\kappa_{1,2} P_{1,2} Y}\right)\right] \\
\text { with } Z \sim \chi_{2 N_{t}}^{2} Y \sim \chi_{2}^{2} \quad \text { if }(\mathrm{BS} 1)=(\mathrm{MRC})
\end{array}\right. \\
Q \mathbb{E}\left[\begin{array}{c}
\left.\log _{2}\left(1+\frac{P_{1,1} \gamma_{11} Z}{1+\kappa_{1,2} P_{1,2} Y}\right)\right]+(1-Q) \mathbb{E}\left[\log _{2}\left(1+\frac{P_{1,1} \gamma_{1,1} Z}{1+P_{1,2} Y}\right)\right] \\
\text { with } Z \sim \chi_{2\left(N_{t}-1\right)}^{2} Y \sim \chi_{2}^{2} \quad \text { if }(\mathrm{BS} 1)=(\mathrm{PZF})
\end{array}\right.
\end{array}\right.
\end{aligned}
$$

where $Q$ is the ratio of the two surfaces $\mathcal{R}_{\text {Inner }} / \mathcal{R}_{\text {cell }}$ assuming a uniform distribution of the users within the cells.

\section{The proposed algorithm for more than 2-cells networks:}

For the multicell networks including more than 2-cells, we share the number of quantized bits for the interferer BSs unequally. The algorithm that performs this bit selection strategy for interferer BSs is described as follows:

- Step $2 b$ :

- Bit selection strategy for interferer BS: We propose to share the remaining number of bits, $B_{\max }-B_{k, k}$, unequally to transfer the CDI of the interferer BSs. The sharing strategy is based on quantizing the information of the dominant interferer using more bits. Therefore, we define the sharing mechanism based on the distance ratio.

For each $k^{\prime}=1,2, \ldots, K$ and $k^{\prime} \neq k$ :

$$
\begin{aligned}
& B_{k, k^{\prime}}=\frac{\left(B_{\max }-B_{k, k}\right)\left(1 / d_{k, k^{\prime}}\right)}{S_{k}} \\
& \text { where } \quad S_{k}=\sum_{\substack{a=1 \\
a \neq k}}^{K}\left(1 / d_{k, a}\right)
\end{aligned}
$$

\section{Simulation RESUlts}

We perform the simulation results to illustrate the benefits of the proposed adaptive feedback link in a cooperative multicell system. For the simulations, we chose the parameters as $N_{t}=$ 4, $R=1 \mathrm{~km}$ and $\alpha=3.7$.

Depending on the values of $P_{0}$, we can select to perform the different transmission strategies in 2-cells networks [6] as only the MRC (For $P_{0}$ is below $10 \mathrm{~dB}$ ), both MRC and PZF (For $P_{0}$ is around 10dB) and only the PZF (For $P_{0}$ is above $10 \mathrm{~dB})$. 
Firstly, we consider a simplified 2-cells network model where the positions of $\mathrm{BS} 1$ and $\mathrm{BS} 2$ are $(-R, 0)$ and $(+R, 0)$ respectively.

In Figure 2, we show the required number of bits to maximize the sum data rate of the 2-cells network at $P_{0}=10 \mathrm{~dB}$. It is observed that the number of bits allocated bits for the serving and interferer BSs only depends on the location of the user itself in the 2-cells network and does not change according to the location of the other user in the neighboring cell. In addition to that, both MRC and PZF transmission strategies can be performed at the BSs considering the number of allocated bits for serving and interfering BSs. According to this bit partitioning scheme, the inner and outer regions can be determined as

$$
\begin{aligned}
\mathcal{R}_{\text {Inner }} & =\left\{0.45 R \leq\left|x_{k, k}\right| \leq R\right\} \\
\mathcal{R}_{\text {Outer }} & =\left\{R<\left|x_{k, k}\right|<0.45 R\right\}
\end{aligned}
$$

with $k=1,2$. In addition to that, we can define two subregions ( $L=2)$ as,

$$
\begin{aligned}
& \mathcal{R}_{\mathrm{PZF}_{1}}=\left\{0.25 R \leq\left|x_{k, k}\right|<0.45 R\right\}, \quad B_{k, k}=5 \\
& \mathcal{R}_{\mathrm{PZF}_{2}}=\left\{0 \leq\left|x_{k, k}\right|<0.25 R\right\}, \quad B_{k, k}=4
\end{aligned}
$$

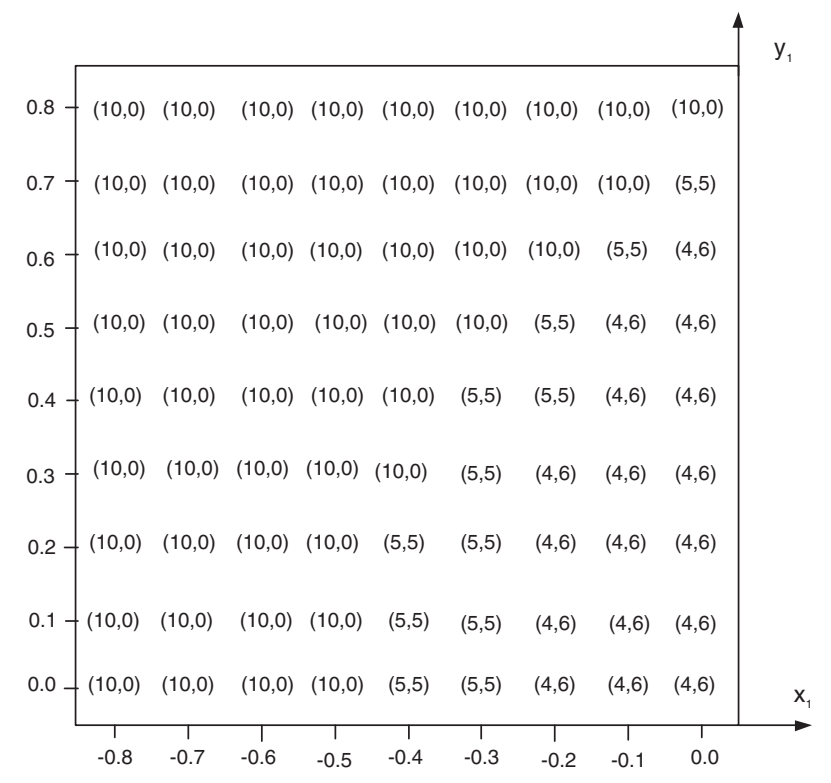

Fig. 2. Optimal bit partitioning for User 1 at $P_{0}=10 \mathrm{~dB}$ for 2-cells network.

In Figure 3, we compare the proposed algorithm with the scheme where only the PZF is applied at the BS by sharing the quantization bits for serving and interfering BSs equally. We observe that the proposed algorithm improves the sum capacity by choosing the bit partitioning scheme and the best transmission strategy according to the location of the user in 2-cells network and the defined regions.

Assuming the users are uniformly distributed in the cell area, we evaluate the performance of the proposed algorithm in 2-cells network at $P_{0}=10 \mathrm{~dB}$ considering the regions and subregions defined in (11) and (12). We compare the proposed

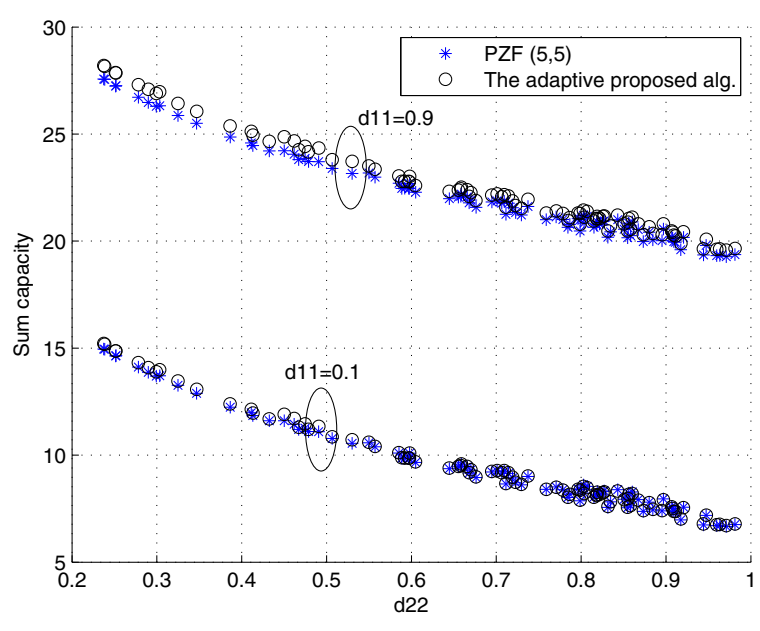

Fig. 3. The sum capacity versus the location of the users at $P_{0}=10 \mathrm{~dB}$ 2-cells network.

algorithm with the scheme that only the MRC is employed with $B_{1,1}=B_{2,2}=B_{\max }=10$. According to the sum capacity results as shown in Figure 4 , the proposed algorithm improves the sum capacity with the percentage of $4-16 \%$ compared to only the MRC scheme by adaptively selecting the transmission and bit partitioning strategy depending only on the users' itself location in 2-cells network.

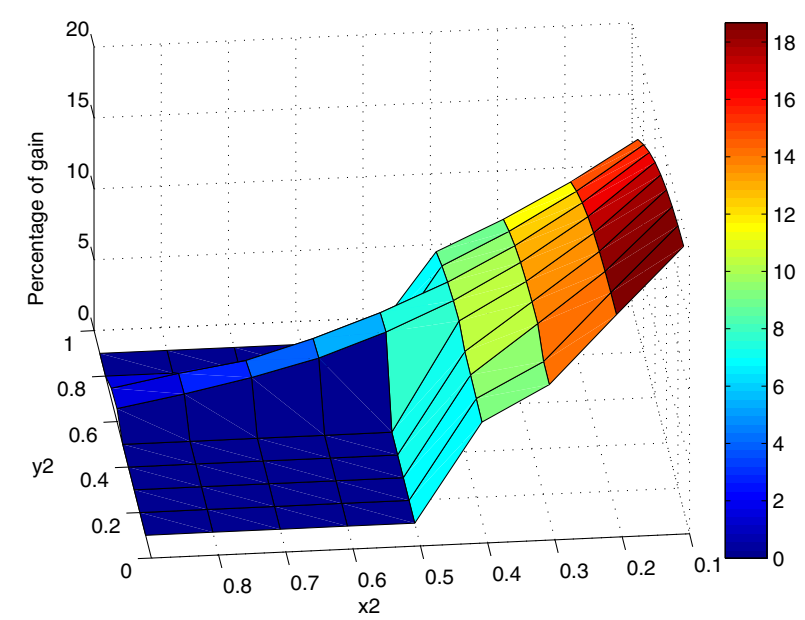

Fig. 4. The performance gain between MRC and the proposed algorithm at $P_{0}=10 \mathrm{~dB}$ for 2-cells network.

In order to illustrate the performance of multicell networks, we simulate 3-cells network employing the proposed adaptive algorithm. Firstly, we obtain the optimum bit partitioning to maximize the sum capacity for both the serving and the interferer BSs as drawn in Figure 5 according to the location of the user 1 for $B_{\max }=30$ bits. The same kind of bit partitioning scheme is also obtained for the other users belonging to their locations in 3-cells network.

Considering the optimal case, we can find the predefined 


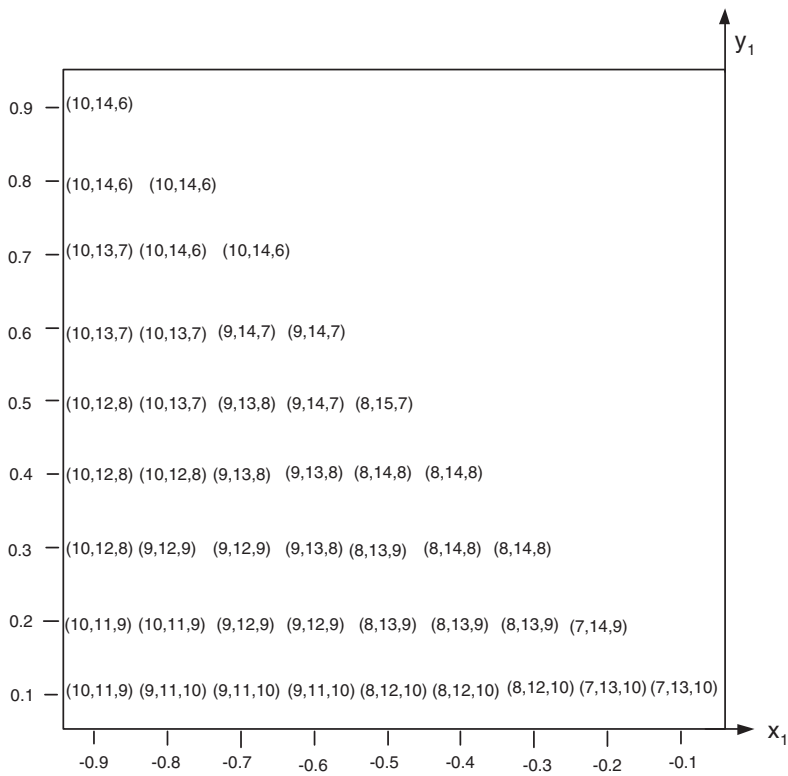

Fig. 5. Optimal bit partitioning for User 1 as $\left(B_{1,1}, B_{1,2}, B_{1,3}\right)$ at $P_{0}=$ $5 \mathrm{~dB}$ for 3-cells networks.

bits and corresponding subregions as follows:

$$
\begin{aligned}
& \mathcal{R}_{\mathrm{PZF}_{1}}=\left\{0.85 R \leq\left|x_{k, k}\right| \leq R\right\}, \quad B_{1,1}=10 \\
& \mathcal{R}_{\mathrm{PZF}_{2}}=\left\{0.55 R \leq\left|x_{k, k}\right|<0.85 R\right\}, \quad B_{1,1}=9 \\
& \mathcal{R}_{\mathrm{PZF}_{3}}=\left\{0.25 R \leq\left|x_{k, k}\right|<0.55 R\right\}, \quad B_{1,1}=8 \\
& \mathcal{R}_{\mathrm{PZF}_{4}}=\left\{0 \leq\left|x_{k, k}\right|<0.25 R\right\}, \quad B_{1,1}=7
\end{aligned}
$$

In Figure 6, we evaluate the performance of the proposed algorithm in a 3-cells network for $B_{\max }=36$ and different values of $P_{0}$ comparing by the MRC only scheme and the PZF only scheme with an uniform bit partitioning of $(12,12,12)$. According to the sum capacity results, it is shown that the proposed algorithm improves the performance of only the PZF scheme with uniform bit allocation by $7-12 \%$ in average. At the cell edges, the performance gain increases to $11-16 \%$.

\section{CONCLUSION}

In this paper, we have considered interference cancelation strategies in cooperative downlink multicell systems with limited feedback link. In order to achieve sum capacity improvement on the multicell network, we have employed two classical beamforming strategies for the design of the beamforming vectors: maximum ratio combining and partial zero-forcing beamforming. We have shown that the required bit partitioning for the transmission strategies can be chosen according only to the users'location and the degree of the received power at the edge of the cells. We have proposed a simple adaptive bit partitioning strategy by defining optimal regions and subregions adaptively depending on the location of the user in the multicell network. We have shown that the proposed algorithm having a simple structure for both users and base stations improves the sum capacity of $16 \%$ in the cell edges at high $P_{0}$ values for 3-cells networks.

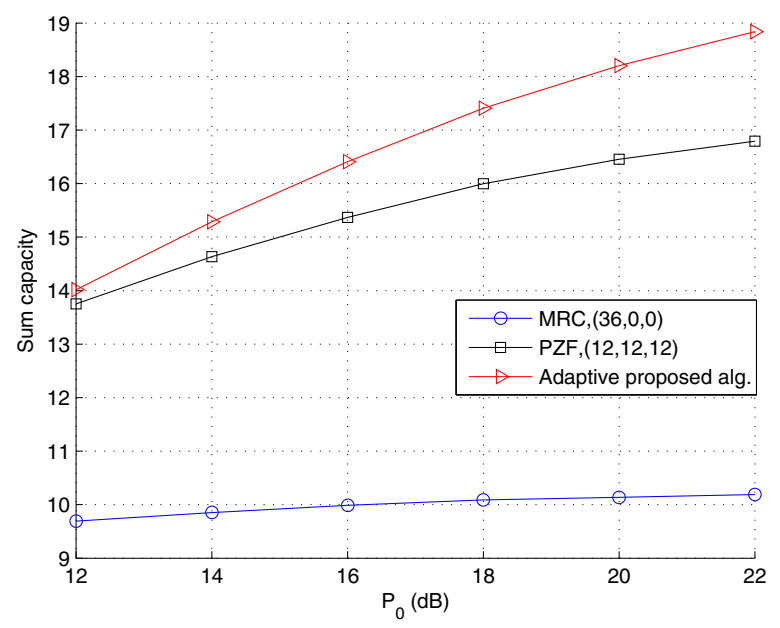

Fig. 6. The performance of different strategies versus $P_{0}$ in a 3-cell network

\section{ACKNOWLEDGMENT}

This research was supported by a Marie Curie Intra European Fellowship within the 7th European Community Framework Programme as a part of INTERCELL project with the contract number PIEF-GA-2009-255128.

\section{REFERENCES}

[1] M. Karakayali, G. Foschini, and R. Valenzuela, "Network coordination for spectrally efficient communications in cellular systems", IEEE Wireless Communications Magazine, vol. 13, no. 4, pp. 56-61, Aug. 2006.

[2] H. Zhang and H. Dai, "Cochannel interference mitigation and cooperative processing in downlink multicell multiuser MIMO networks", EURASIP Journal on Wireless Communications and Networking, no. 2, pp. 222-235, 4th Quarter 2004.

[3] S. Shamai (Shitz), O. Somekh, and B. M. Zaidel, "Multi-cell communications: An information theoretic perspective", Joint Workshop on Communications and Coding (JWCC), Florence, Italy, Oct. 2004.

[4] F. Boccardi and H. Huang, "Limited downlink network coordination in cellular networks", Proc. of the IEEE Int. Symp. on Personal Indoor and Mobile Radio Comm. (PIMRC), Athens, Greece, Sept. 2007.

[5] N. Jindal, J. G. Andrews, and S. Weber, "Rethinking MIMO for wireless networks: Linear throughput increases with multiple receive antennas", Proc., IEEE Intl. Conf. on Communications (ICC), Dresden, Germany, Jun. 2009.

[6] J. Zhang and J. G. Andrews, "Adaptive Spatial Intercell Interference Cancellation in Multicell Wireless Network", submitted to Transaction on Information Theory, sept. 2009, arxiv preprint arXiv:0909.2894.

[7] R. Bhagavatula, R. W. Heath, Jr., "Sum-Rate Maximizing Beamforming in Multicell Systems with Limited Feedback", Proc. of the IEEE Asilomar Conf. on Signals, Systems, and Computers, Pacific Grove, CA, pp. 1838-1842, November 1-4, 2009.

[8] L. Liu, J.(C.) Zhang, J.-C. Yu, J. Lee, "Intercell Interference Coordination through Limited Feedback", Eurasip International Journal of Digital Multimedia Broadcasting, Volume 2010, Article ID 134919, doi:10.1155/2010/134919.

[9] A. Narula, M. J. Lopez, M. D. Trott and G. W. Wornell, "Efficient use of side information in multiple antenna data transmission over fading channels", IEEE Journal on Sel. Areas in Commun. (JSAC), pp.14231436, Oct. 1998

[10] C. K. Au-Yeung and D. J. Love,"Performance Analysis of Random Vector Quantization Limited Feedback Beamforming”, Proc. of IEEE Asilomar Conf. on Signals, Systems, and Computers, Pacific Grove, CA, nov. 2005 .

[11] N. Jindal, "MIMO broadcast channels with finite-rate feedback", IEEE Transactions on Information Theory, vol. 52, no.11, pp. 50455060, 2006. 